\title{
Applications of Thin Insulating Film-Coated Electrodes for Hot Electron Injection into Aqueous Electrolyte Solutions
}

\author{
Dr. Sakari Kulmala*, Kalle Salminen, and Päivi Kuosmanen
}

\begin{abstract}
Hot electrons can be injected into aqueous electrolyte solution from tunnel emission electrodes which are composed of a conductor coated with a thin insulating film. Conductors can be e.g. metals, or strongly doped semiconductors $[1,2]$. Insulating film should be fabricated from an insulator material with a band gap of ca. $5 \mathrm{eV}$ or preferably higher, and the Fermi level of the insulator should be somewhere in the mid band gap region. The hot electrons are emitted to aqueous electrolyte solution by direct field-assisted tunneling $[2,3]$. After injection into the conduction band of water the hot electrons are thermalized and solvated to form hydrated electrons which finally act as reducing mediators in the Hot Electron-induced Electrochemiluminescence (HECL) systems. HECL is utilized mainly in immunoanalysis and in DNA-probe assays in which electrochemiluminescent labels are utilized [ $3-5 \mathrm{crp}, 2$, tsh]. Best labels are aromatic lanthanide chelates which allow time-resolved HECL detection [3, 6].

Best results have been obtained with silicon electrodes or silicon chips on which the working electrode/electrodes and counter electrode have been integrated [5,7]. However, a low-cost alternative is sputtered or vacuum evaporated aluminum whose surface has been thermally oxidized at room temperature in room air. Recently, we have developed printable tunnel emission electrodes for disposable plastic assay cartridges, but we are not allowed to disclose much of that research at the moment.
\end{abstract}

\section{References}

[1] S. Kulmala, A. Kulmala, T. Ala-Kleme, J. Pihlaja, Primary cathodic steps of electrogenerated chemiluminescence of lanthanide(III) chelates at

oxide-covered aluminum electrodes in aqueous solution, Anal. Chim. Acta. 367 (1998) 17-31.

[2] S. Kulmala, H. Joela, T. Ala-Kleme A. Kulmala, Hot electron injection into aqueous electrolyte solution from thin insulating film-coated electrodes,

J. Radioanal and Nucl. Chem. 232 (1998) 91-95.

[3] J. Suomi, S. Kulmala, Hot Electron-Induced Electrogenerated Chemiluminescence, in: Rev. Fluoresc. 2009, 2011: pp. 47-73.

[4] T. Ala-Kleme, P. Makinen, T. Ylinen, L. Vare, S. Kulmala, P. Ihalainen, et al., Rapid electrochemiluminoimmunoassay of human $\mathrm{C}$-reactive protein

at planar disposable oxide-coated silicon electrodes, Anal. Chem. 78 (2006) 82-88.

[5] T. Ylinen-Hinkka, a. J. Niskanen, S. Franssila, S. Kulmala, Immunoassay of C-reactive protein by hot electron induced electrochemiluminescence

using integrated electrodes with hydrophobic sample confinement, Anal. Chim. Acta. 702 (2011) 45-49..

[6] S. Kulmala, T. Ala-Kleme, J. Suomi, Electrochemiluminescence of Lanthanides, in: Springer Series, Lanthan. Lumin., 2011: pp. 343-360.

[7] Antti J. Niskanen, Tiina Ylinen-Hinkka, Matti Pusa, Sakari Kulmala, Sami Franssila,

Dr. Sakari Kulmala*, Kalle Salminen, and Päivi Kuosmanen

Prof. of Analytical Chemistry

Aalto University

Finland 